\title{
Inhibition of inducible nitric oxide synthase (iNOS) by simvastatin attenuates cardiac hypertrophy in rats
}

\author{
A.M. Ahmed \\ Department of Anatomy, College of Medicine, King Saud University, Riyadh, Saudi Arabia \\ Received: 7 April 2016; Accepted: 19 May 2016]
}

Background:The left ventricular hypertrophy (LVH) occurs in response to the haemodynamic overload in some physiological and pathological conditions. This study was designed to investigate the possible cardioprotective effect of simvastatin (SIM) treatment against isoproterenol (ISO)-induced LVH and the probable underlying mechanism in adult male Wistar rats.

Materials and methods: Animals were allocated into four groups. Rats of control group received normal saline orally for 30 days and intraperitoneally for the last 7 days. Rats of SIM group received SIM orally (10 mg/kg/day in saline) for 30 days. Rats of ISO group received normal saline orally for 30 days and ISO intraperitoneally $(5 \mathrm{mg} / \mathrm{kg}$ ) for the last 7 days to induce $L V H$. Rats of ISO/SIM group received SIM for 30 days and ISO intraperitoneally for the last 7 days. At the end of the experiment, all animals were sacrificed by cervical decapitation under anaesthesia. Truncal blood was collected and serum was separated and used for biochemical assay. The heart was dissected and processed for histological and immunohistochemical studies.

Results: The results of the present study confirmed the ISO-induced myocardial lesions including significant increase of heart weight (HW), heart weight/body weight (HW/BW) ratio, LVH, interstitial myocardial fibrosis (increased collagen types I and III), inflammatory cellular infiltration, necrosis of cardiomyocytes, and increased expression of inducible nitric oxide synthase (iNOS) and thioredoxin in cardiomyocytes. These changes were accompanied by significant increase in serum levels of troponin-T, creatine phosphokinase-MB (CPK-MB), tumour necrosis factor-alpha (TNF- $\alpha$ ) and interleukin-6 (IL-6). Co-administration of SIM to ISO-injected rats significantly reduced all these cardiac changes and serum biochemical markers in addition to marked depletion of iNOS and thioredoxin expression in cardiomyocytes.

Conclusions: It is concluded that SIM co-administration attenuated ISO-induced cardiac lesions including LVH by inhibiting iNOS expression in cardiomyocytes. (Folia Morphol 2017; 76, 1: 15-27)

Key words: left ventricular hypertrophy, isoproterenol, simvastatin, inducible nitric oxide synthase, thioredoxin, collagen, rats, immunohistochemistry

Address for correspondence: A.M. Ahmed, Assiociate Professor, Department of Anatomy, College of Medicine, King Saud University, PO Box 2925 (28), Riyadh 11461, Saudi Arabia, tel: +966(11)4670809, fax: +966(11)4671300, e-mail: alymahmed53@gmail.com 


\section{INTRODUCTION}

Hypertension is one of the main causes of left ventricular cardiac hypertrophy (LVH) that is considered as the major risk factor for heart failure $[16,32,56]$. The key pathological processes in LVH include hypertrophy of the cardiomyocytes and fibrosis of the myocardial interstitium [24]. Failure of the hypertrophied heart involves a complex sequence of events at the molecular and cellular levels including alteration in the metabolism of extracellular matrix collagen [40]. Porter and Turner [40] reported that extracellular matrix collagen plays an essential role in maintaining the architecture and preserving the contractile function of the myocardium. It was found that a large amount of collagen is secreted into the intercellular spaces of the myocardium during the process of LVH [21]. This contributes to myocardial stiffness and ventricular dysfunction [50]. It was hypothesized that enhancement of myocardial fibrosis secondary to increased collagen types I and III synthesis and deposition leads to development of heart failure in cases with hypertensive heart disease $[24,41]$.

Inducible nitric oxide synthase (iNOS) is normally expressed at a low level in cardiomyocytes [44]. Several investigators have demonstrated that iNOS expression in cardiomyocytes is increased by numerous stimuli including inflammatory mediators, cytokines, tissue ischaemia, hypertension, and LVH [19, 31, $38,44]$. Overexpression of iNOS in cardiomyocytes increased myocardial nitric oxide (NO) production and resulted in inflammatory cell infiltrate, LVH, cardiac fibrosis and contractile dysfunction. Moreover, iNOS deficiency (iNOS ${ }^{--}$) attenuates LVH and iNOS expressed in response to systolic overload serves as a source for myocardial reactive oxygen species (ROS) that contribute to LVH [61].

Induction of iNOS produces excessive amounts of $\mathrm{NO}$, which reacts with superoxide $\left(\mathrm{O}_{2}^{-}\right)$to form the high cytotoxic oxidant peroxynitrite. Also, iNOS itself is capable to produce $\mathrm{O}_{2}^{-}$, which is transformed to hydrogen peroxide $[13,23,59]$. Both peroxynitrite and hydrogen peroxide induced tissue injury and organ dysfunction including the heart $[18,23,36]$.

Oxidative stress produced by ROS is known to regulate collagen metabolism in cardiac fibroblasts, which are the major cell type responsible for collagen synthesis and degradation in the myocardium [46]. In addition, ROS may be involved in triggering inflammatory reactions through the induction of cytokines [29]. Previous investigations $[7,43]$ have demonstrated that repeated daily injection of isoproterenol (ISO) induces substantial LVH, necrosis, and fibrosis. These changes are attributed to an increase in myocardial oxidative stress as a result of the formation of the highly cytotoxic oxidant peroxynitrite [39]. Also, experimental data suggest that the release of $\mathrm{O}_{2}{ }^{-}$induces cardiac myocyte hypertrophy [51]. Inhibiting LVH has emerged as a primary target for therapeutic approaches in preventing hypertension-induced end-organ damage [35].

Statins, hydroxymethylglutaryl-coenzyme A reductase inhibitors, are widely prescribed cholesterol-lowering agents that decrease the incidence of myocardial infarction and ischaemic stroke [26]. Simvastatin (SIM), a member of statins, has been recognised to have antioxidant effects and is an effective agent for preventing the development of cardiac hypertrophy (through an antioxidant mechanism) [51]. Hence, the present study was designed to investigate the possible anti-hypertrophic activity of SIM pretreatment against ISO-induced LVH in rats and to determine the potential underlying mechanism involved.

\section{MATERIALS AND METHODS}

\section{Experimental animals}

Twenty-four adult male Wistar albino rats, with a body weight (BW) between 160 and $180 \mathrm{~g}$, were supplied by the Animal Care Centre at the College of Medicine, King Saud University, Riyadh, Kingdom of Saudi Arabia. Rats were housed and maintained under standard controlled environmental conditions in individual plastic cages at a temperature of $25 \pm 2^{\circ} \mathrm{C}$ on 12-h light/dark cycle throughout the study with free access to standard pelleted rat chow and tap water ad libitum. The animals were acclimatised for a period of 1 week before the start of the experiment. All the experimental procedures were conducted according to the Guidelines for the Care and Use of Laboratory Animals of the College of Medicine Research Centre (CMRC) at King Saud University and conform to the Guide for the Care and Use of Laboratory Animals published by the National Institutes of Health (NIH).

\section{Chemicals}

Isoproterenol and simvastatin were purchased from Sigma, St. Louis, Mo, USA. Mouse monoclonal anti-iNOS (anti-NOS-2) antibody was obtained from Santa Cruz Biotechnology (Dallas, TX, USA). Mouse monoclonal anti-collagen III antibody and rabbit polyclonal anti-thioredoxin were obtained from abcam (Cambridge, UK). SIM is one of hydroxymethylglutaryl- 
-coenzyme A reductase inhibitors. It has antioxidant properties [38].

\section{Experimental design}

Animals were randomly allocated into four groups of 6 rats each:

- Control group: rats received normal saline orally for 30 days and intraperitoneally for the last 7 days;

- SIM group: rats received SIM (10 mg/kg/day) dissolved in saline by oral gavage for 30 days [8];

- ISO group: rats received normal saline orally for 30 days and ISO ( $5 \mathrm{mg} / \mathrm{kg} /$ day intraperitoneally) for the last 7 days $[57,60]$;

- ISO/SIM group: rats received SIM $(10 \mathrm{mg} / \mathrm{kg})$ dissolved in saline by oral gavage for 30 days and ISO ( $5 \mathrm{mg} / \mathrm{kg} /$ day intraperitoneally) for the last 7 days. At the end of the experiment, all animals were sacrificed by cervical decapitation under anaesthesia and truncal blood samples were collected and centrifuged to separate sera. Sera were kept at $-20^{\circ} \mathrm{C}$ and used for biochemical assays. Hearts were immediately excised, fixed in $10 \%$ buffered formalin and processed for histological and immunohistochemical studies.

Body weight, heart weight, and heart weight/ /body weight ratio

At the end of the experiment, BW and heart weight $(\mathrm{HW})$ were measured. The HW/BW ratio was calculated and used as a sensitive indicator of cardiac hypertrophy $[15,58]$.

\section{Serum biochemical analysis}

Determination of serum levels of troponin-T and creatine phosphokinase-MB (CPK-MB). Serum levels of troponin-T were measured using a Siemens Dimension Xpand Plus instrument (IL, USA). Also, serum levels of CPK-MB isoenzyme were determined with an auto-analyser (ILab-300, bioMérieux Diagnostics, Milan, Italy). Both troponin-T and CPK-MB are cardiac injury markers. CPK-MB is the bound combination of two variants (isoenzymes, CPKM and CPKB) of the enzyme creatine phosphokinase [17].

Determination of serum levels of tumour necrosis factor-alpha (TNF- $\alpha$ ). The serum concentration of TNF- $\alpha$ (an inflammatory cytokine) was measured using enzyme-linked immunosorbent assay (ELISA) kits following the instructions supplied by the manufacturer (DuoSet Kits, R\&D Systems, Minneapolis, MN, USA).

Determination of serum levels of interleukin-6 (IL-6). Serum levels of IL-6 were measured by ultra- sensitive ELISA (Quantikine HS Human IL-6 Immunoassay, R\&D Systems, Minneapolis, MN, USA) [25].

\section{Histopathological study}

The excised hearts were fixed in $10 \%$ buffered formalin at $4^{\circ} \mathrm{C}$ for $24 \mathrm{~h}$ and processed to prepare transverse, midventricular, $5-\mu \mathrm{m}$-thick paraffin sections. These sections were stained with haematoxylin and eosin (H\&E) [48] and Masson's trichrome stains [6].

\section{Immunohistochemical study}

Immunostaining of the heart sections for detection of collagen type III, iNOS (NOS-2) and thioredoxin were performed using streptavidin-biotinylated horse radish peroxidase method (Novalink Max Polymer detection system; Novocastra Laboratories, Newcastle, UK). The procedure involved the following steps: endogenous peroxidase activity was inhibited by $3 \% \mathrm{H}_{2} \mathrm{O}_{2}$ in distilled water for $5 \mathrm{~min}$ and then the sections were washed in Tris-buffered saline (TBS, $\mathrm{pH}$ 7.6) (Sigma-Aldrich) for $10 \mathrm{~min}$. Non-specific binding to antibodies was blocked by incubation with protein block for $5 \mathrm{~min}$ (Novocastra). Sections were incubated with an antibody against collagen III (1:100 dilution, abcam, Cambridge, UK), an antibody against iNOS (NOS-2) (1:100 dilution, Santa Cruz, TX, USA) and an antibody against thioredoxin (1:200 dilution, abcam, Cambridge, UK) according to the manufacturer's instructions. Sections were washed 3 times in TBS, each for $3 \mathrm{~min}$, and then incubated with biotinylated IgG (Novocastra) for $30 \mathrm{~min}$, followed by washing in TBS and incubation with Novolink polymer (Novocastra) for $30 \mathrm{~min}$. Sections were washed in TBS for 3 times, each for $3 \mathrm{~min}$. Peroxidase was detected with working solution of diaminobenzidine (DAB) substrate (Novocastra) for $10 \mathrm{~min}$. Sections were washed in distilled water for 10 min, nuclei were stained with Mayer's haematoxylin and sections were mounted in DPX (Dysterene, Plasticizer, Xylene). For negative control sections, the same procedure was followed but with omission of incubation in the primary antibodies.

\section{Image analysis}

High-resolution whole-slide digital scans of all stained sections were created with a ScanScope scanner (Aperio Technologies, Inc.). The digital slide images were then viewed and analysed using the viewing and image analysis tools of Aperio's ImageScope software (Aperio Technologies, Inc.). To 
Table 1. Relative heart weight (heart/body weight; mean \pm standard error of the mean [SEM]) in the studied groups

\begin{tabular}{lcccc}
\hline & Group I (Control) & Group II (SIM) & Group III (ISO) & Group IV (ISO/SIM) \\
\hline Mean \pm SEM & $0.003 \pm 0.000$ & $0.003 \pm 0.000$ & $0.006 \pm 0.000$ & $0.004 \pm 0.000$ \\
P1 & & 0.922 & $0.004^{*}$ & $0.026^{*}$ \\
P2 & 0.922 & & $0.005^{*}$ & $0.001^{*}$ \\
P3 & $0.004^{*}$ & $0.005^{*}$ & & $0.010^{*}$ \\
\hline
\end{tabular}

P1 vs. group I; P2 vs. group II; P3 vs. group III; *significant difference $(\mathrm{p} \leq 0.05)$

Table 2. Left ventricular wall thickness $(\mu \mathrm{m}$; mean \pm standard error of the mean [SEM]) in the studied groups

\begin{tabular}{lcccc}
\hline & Group I (Control) & Group II (SIM) & Group III (ISO) & Group IV (ISO/SIM) \\
\hline Mean \pm SEM & $2.436 \pm 0.082$ & $2.418 \pm 0.091$ & $4.142 \pm 0.116$ & $3.202 \pm 0.134$ \\
P1 & & 1.000 & $0.000^{*}$ & $0.000^{*}$ \\
P2 & 1.000 & & $0.000^{*}$ & $0.000^{*}$ \\
P3 & $0.000^{*}$ & $0.000^{*}$ & & $0.000^{*}$ \\
\hline
\end{tabular}

P1 vs. group I; P2 vs. group II; P3 vs. group III; ${ }^{*}$ significant difference $(p \leq 0.05)$

quantify the immunopositive reaction and the extent of fibrosis, five areas, each with the fixed size of $0.264 \mathrm{~mm}^{2}$, were randomly selected per section, and the colour deconvolution (colour separation) algorithm (Aperio Technologies, Inc.) was set up (by colour calibration) to detect and quantify only the brown colour of DAB positive immunostaining or only the green colour of fibrous tissue (as stained by Masson's trichrome). The algorithm was then run on the selected areas to measure the percentage of the colour of interest relative to the total area of analysis. The thickness of the wall of the left ventricle (LV) was measured on the digital scans of H\&E-stained slides, using the linear measurement tool of Aperio's ImageScope software (Aperio technologies, Inc.). To minimise error, thickness was measured at 5 randomly chosen points per heart section and averages were calculated. All image analysis output results were finally exported to Excel sheets and subjected to statistical analysis.

\section{Statistical analysis}

Data collected were subjected to statistical analysis using IBM SPSS Statistics version 22 software. The homogeneity of variance was first checked with Levene test. Analysis of variance (ANOVA) was used for an overall comparison between the groups followed by Bonferroni test (when the homogeneity of variance assumption was met) or by Games-Howell test (when the homogeneity of variance assumption was not met) as post-hoc tests for pairwise comparisons. Differences were considered significant when $p$ was less than or equal to 0.05 .

\section{RESULTS}

\section{Clinical observations}

All rats used in the present study survived till the end of the experiment. This indicated that intraperitoneal injection of ISO provided a simple, non-invasive procedure to induce myocardial hypertrophy including LVH with almost no incidence of mortality. All animals of ISO group demonstrated lethargy and marked reduction of movements.

\section{Heart weight and heart weight/body weight ratio \\ The results showed significant increase in $\mathrm{HW}$ and $\mathrm{HW} / \mathrm{BW}$ ratio in the ISO-injected rats as compared with the control rats. Treatment of ISO-injected rats with SIM induced significant reduction of both $\mathrm{HW}$ and HW/ /BW ratio as compared with ISO-injected rats (Table 1).}

\section{Left ventricular wall thickness}

Isoproterenol-injected rats exhibited significant increase in LV wall thickness inducing LVH as compared with control rats. Treatment of ISO-injected rats with SIM induced marked decrease in LV wall thickness as compared with ISO-injected rats (Table 2).

\section{Serum biochemical analysis}

Serum levels of troponin-T and CPK-MB. ISO-injected rats exhibited significant increase in serum levels of cardiac injury markers, namely troponin-T and $\mathrm{CPK}-\mathrm{MB}$, as compared with control rats. Treatment of ISO-injected rats with SIM significantly 
Table 3. Serum troponin T levels (pg/mL; mean \pm standard error of the mean [SEM]) in the studied groups

\begin{tabular}{lcccc}
\hline & Group I (Control) & Group II (SIM) & Group III (ISO) & Group IV (ISO + SIM) \\
\hline Mean \pm SEM & $7.196 \pm 0.341$ & $7.198 \pm 0.271$ & $48.750 \pm 0.649$ & $25.784 \pm 0.654$ \\
P1 & & 1.000 & $0.000^{*}$ & $0.000^{*}$ \\
P2 & 1.000 & & $0.000^{*}$ & $0.000^{*}$ \\
P3 & $0.000^{*}$ & $0.000^{*}$ & & $0.000^{*}$ \\
\hline
\end{tabular}

P1 vs. group I; P2 vs. group II; P3 vs. group III; *significant difference ( $p \leq 0.05)$

Table 4. Serum creatine phosphokinase-MB levels (U/L; mean \pm standard error of the mean [SEM]) in the studied groups

\begin{tabular}{lcccc}
\hline & Group I (Control) & Group II (SIM) & Group III (ISO) & Group IV (ISO + SIM) \\
\hline Mean \pm SEM & $33.548 \pm 0.733$ & $33.460 \pm 0.888$ & $195.432 \pm 0.548$ & $119.806 \pm 0.748$ \\
P1 & & 1.000 & $0.000^{*}$ & $0.000^{*}$ \\
P2 & 1.000 & & $0.000^{*}$ & $0.000^{*}$ \\
P3 & $0.000^{*}$ & $0.000^{*}$ & & $0.000^{*}$ \\
\hline
\end{tabular}

P1 vs. group I; P2 vs. group II; P3 vs. group III; ${ }^{*}$ significant difference $(p \leq 0.05)$

Table 5. Serum tumour necrosis factor-alpha levels (pg/mL; mean \pm standard error of the mean [SEM]) in the studied groups

\begin{tabular}{lcccc}
\hline & Group I (Control) & Group II (SIM) & Group III (ISO) & Group IV (ISO + SIM) \\
\hline Mean \pm SEM & $7.012 \pm 0.477$ & $6.900 \pm 0.341$ & $34.888 \pm 2.160$ & $16.394 \pm 0.606$ \\
P1 & & 1.000 & $0.000^{*}$ & $0.000^{*}$ \\
P2 & 1.000 & & $0.000^{*}$ & $0.000^{*}$ \\
P3 & $0.000^{*}$ & $0.000^{*}$ & & $0.000^{*}$ \\
\hline
\end{tabular}

$\mathrm{P} 1$ vs. group I; $\mathrm{P} 2$ vs. group II; $\mathrm{P} 3$ vs. group III; * ${ }^{*}$ significant difference $(\mathrm{p} \leq 0.05)$

Table 6. Serum interleukin- 6 levels ( $\mathrm{pg} / \mathrm{mL}$; mean \pm standard error of the mean [SEM]) in the studied groups

\begin{tabular}{lcccc}
\hline & Group I (Control) & Group II (SIM) & Group III (ISO) & Group IV (ISO/SIM) \\
\hline Mean \pm SEM & $7.778 \pm 0.351$ & $7.636 \pm 0.431$ & $34.336 \pm 0.762$ & $13.332 \pm 0.558$ \\
P1 & & 1.000 & $0.000^{*}$ & $0.000^{*}$ \\
P2 & 1.000 & & $0.000^{*}$ & $0.000^{*}$ \\
P3 & $0.000^{*}$ & $0.000^{*}$ & & $0.000^{*}$ \\
\hline
\end{tabular}

P1 vs. group I; P2 vs. group II; P3 vs. group III; *significant difference $(p \leq 0.05)$

reduced the serum levels of these cardiac injury markers as compared with ISO-injected rats (Tables 3,4).

Serum levels of TNF- $\alpha$ and IL-6. Serum levels of proinflammatory biomarkers, namely TNF- $\alpha$ and IL-6, were significantly elevated in ISO-injected rats as compared with control rats and significantly reduced in ISO/SIM rats as compared with ISO-injected rats (Tables 5, 6).

\section{Histopathological study}

Haematoxylin and eosin-stained mid-ventricular cross sections of the heart from control rats and rats treated with SIM alone showed normal microscopic features of cardiomyocytes and interstitial connective tissue (Fig. 1A, B). However, H\&E-stained sections of the heart from ISO-injected rats revealed focal areas of histopathological lesions primarily limited to the wall of the LV as evidenced by degeneration and necrosis of cardiomyocytes, inflammatory cell infiltration, cardiac interstitial fibrosis (Fig. 1C) and an increase in the thickness of LV wall. Simultaneous administration of SIM with ISO injection markedly decreased the histopathological lesions induced by ISO injection alone (Fig. 1D). 

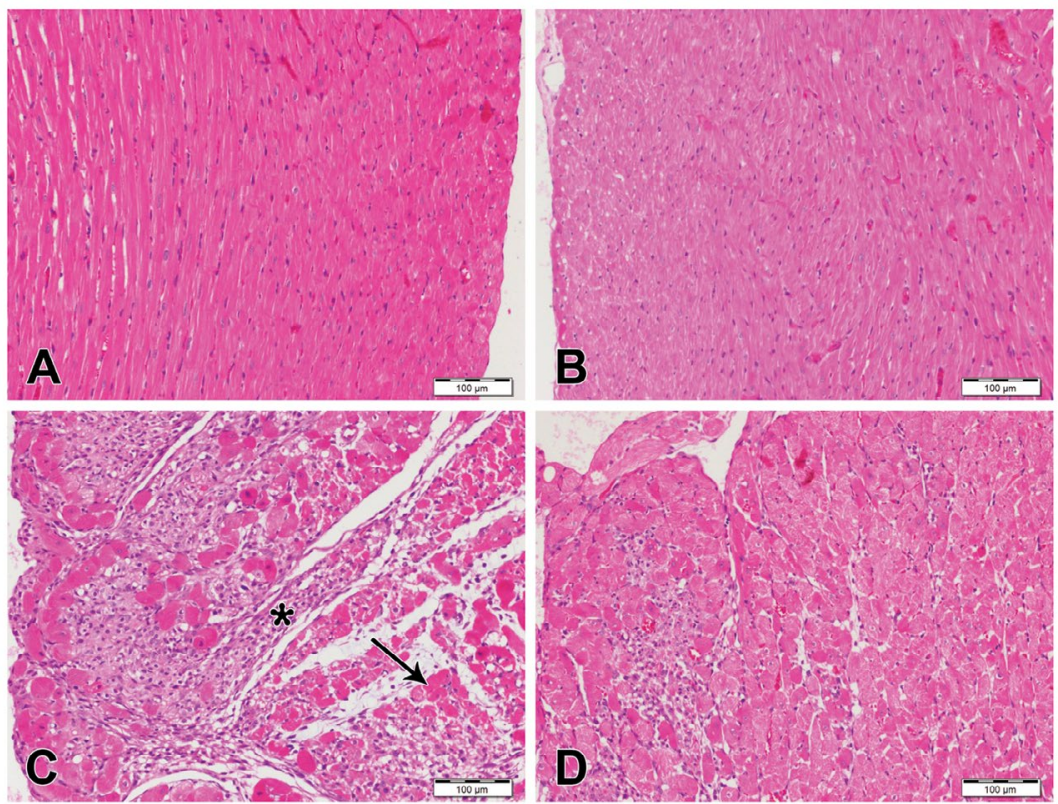

Figure 1. Heart sections stained with haematoxylin and eosin. From control rat (A), and from SIM group (B) showing normal architecture; C. From ISO group showing extensive necrosis of numerous cardiomyocytes (arrow) and inflammatory cell infiltration (asterisk); D. From ISO/SIM group showing marked reduction of histopathological lesions. Scale bars $=100 \mu \mathrm{m}$.
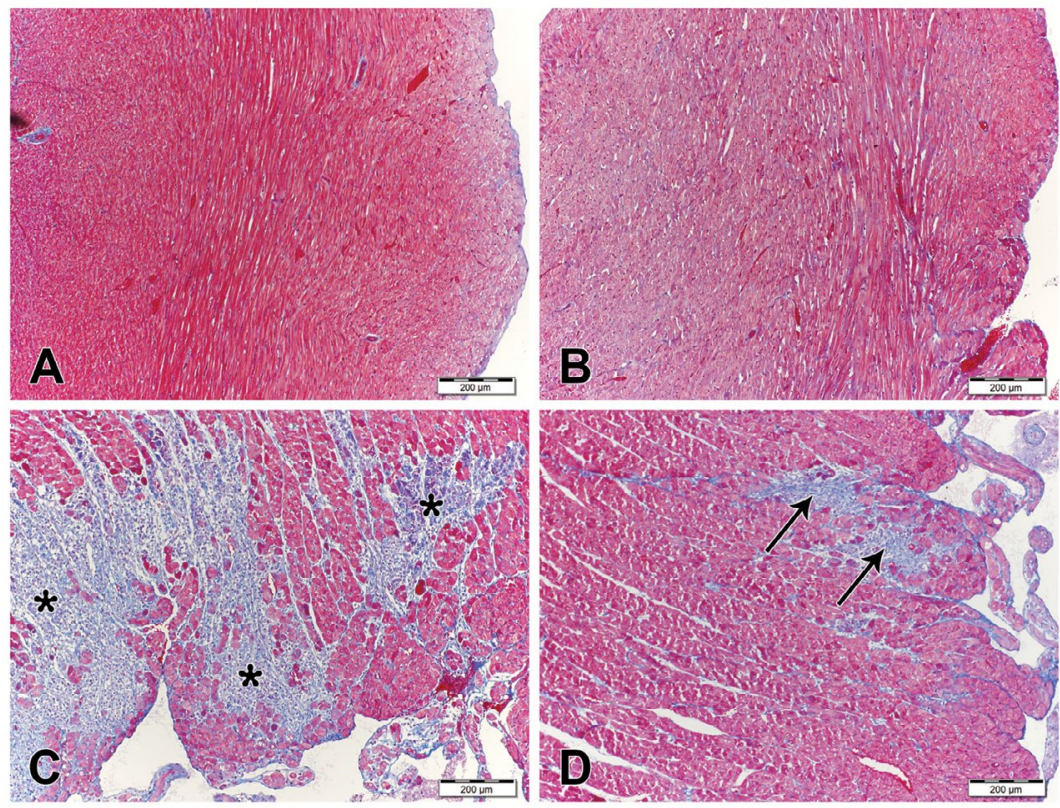

Figure 2. Heart sections stained with Masson's trichrome. From control rat (A), and from SIM group (B) showing scanty fibres of collagen type I; C. From ISO group showing extensive deposition of collagen type I fibres (asterisks); D. From ISO/SIM group showing small patches of collagen type I fibres (arrows). Scale bars $=200 \mu \mathrm{m}$.

With Masson's trichrome stain, mid-ventricular cross sections of the heart from control rats and rats treated with SIM alone demonstrated normal histological appearance and normal collagen architecture with sparsely deposited collagen among the cardiomyocytes (Fig. 2A, B). However,
ISO injection induced a significant increase of the interstitial collagen network in the myocardium (Fig. 2C). On the other hand, co-administration of SIM in ISO-injected rats significantly reduced the interstitial collagen fibrosis (Table 7) as expressed by small patches of fibrosis (Fig. 2D). 
Table 7. Area per cent of fibrosis as detected by Masson's trichrome (mean \pm standard error of the mean [SEM]) in the studied groups

\begin{tabular}{lcccc}
\hline & Group I (Control) & Group II (SIM) & Group III (ISO) & Group IV (ISO/SIM) \\
\hline Mean \pm SEM & $1.810 \pm 0.250$ & $2.060 \pm 0.435$ & $21.286 \pm 2.015$ & $12.013 \pm 3.273$ \\
P1 & & 0.957 & $0.002^{*}$ & 0.112 \\
P2 & 0.957 & & $0.002^{*}$ & 0.119 \\
P3 & $0.002^{*}$ & $0.002^{*}$ & & 0.166 \\
\hline
\end{tabular}

P1 vs. group I; P2 vs. group II; P3 vs. group III; *significant difference $(p \leq 0.05)$

Table 8. Area per cent of collagen-III immunopositivity (mean \pm standard error of the mean [SEM]) in the studied groups

\begin{tabular}{lcccc}
\hline & Group I (Control) & Group II (SIM) & Group III (ISO) & Group IV (ISO/SIM) \\
\hline Mean \pm SEM & $0.141 \pm 0.025$ & $0.279 \pm 0.044$ & $6.177 \pm 0.779$ & $2.301 \pm 0.231$ \\
P1 & & 0.113 & $0.005^{*}$ & $0.002^{*}$ \\
P2 & 0.113 & & $0.006^{*}$ & $0.003^{*}$ \\
P3 & $0.005^{*}$ & $0.006^{*}$ & & $0.021^{*}$ \\
\hline
\end{tabular}

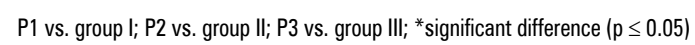

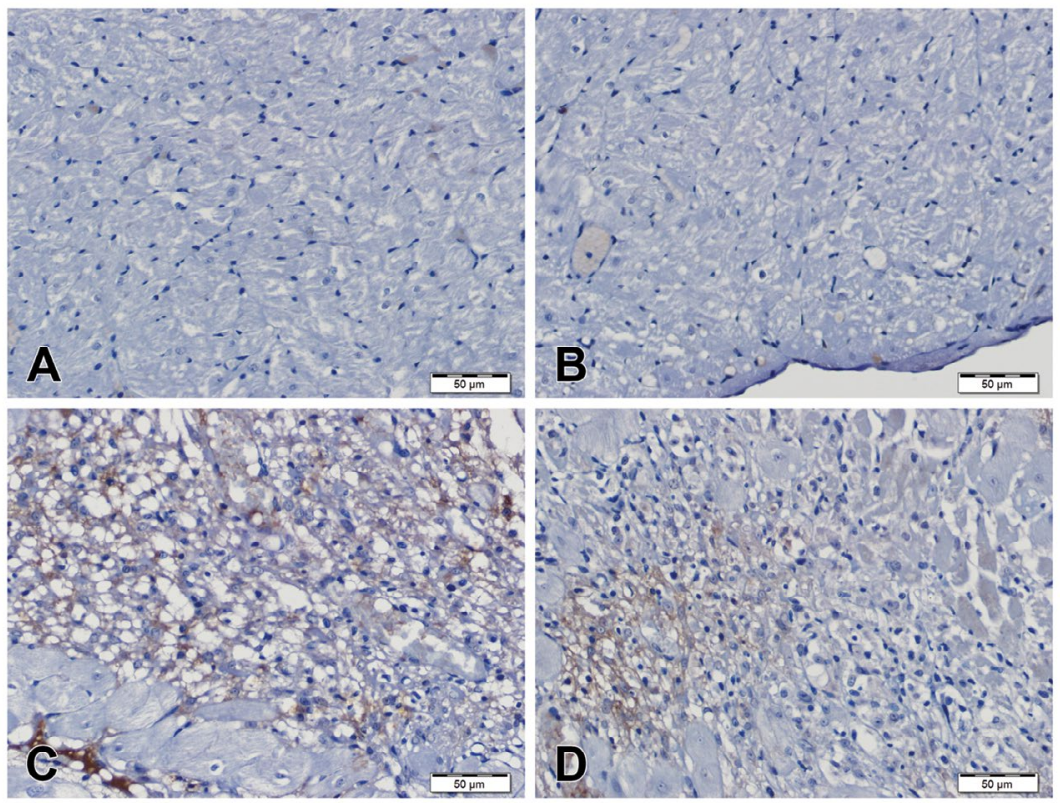

Figure 3. Heart sections immunostained with anti-collagen III antibody. From control rat (A), and from SIM group (B) showing very weak immunoreactivity; C. From ISO group showing considerable immunoreactivity; D. From ISO/SIM group showing mild immunoreactivity. Scale bars $=50 \mu$ m.

\section{Immunohistochemical study}

Immunohistochemical staining of mid-ventricular cross sections of the heart from both control rats and rats treated with SIM alone with anti-collagen III antibody showed sparse deposition of collagen type III fibres in-between cardiomyocytes (Fig. 3A, B). ISO injection induced significant deposition of immunopositive collagen type III fibres extracellularly especially in areas with histopathological lesions (Fig. 3C). Co-administration of SIM with ISO induced significant reduction of deposition of immunopositive collagen type III fibres (Table 8, Fig. 3D).

To assess the myocardial expression of iNOS staining in the different groups, immunohistochemical staining of mid-ventricular sections of the heart with anti-iNOS antibody was performed. The immunostain- 


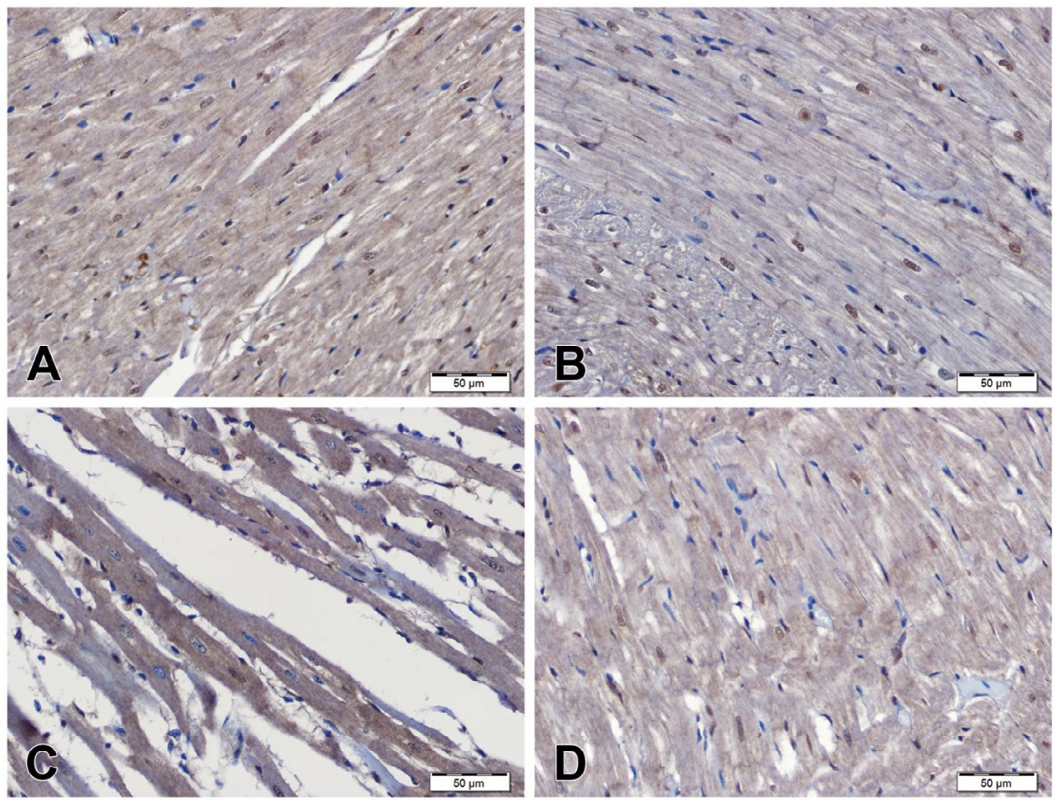

Figure 4. Heart sections immunostained with anti-iNOS antibody. From control rat (A), and from SIM group (B) showing weak immunoreactivity; C. From ISO group showing strong immunoreactivity; D. From ISO/SIM group showing weak immunoreactivity. Scale bars $=50 \mu \mathrm{m}$.

Table 9. Area per cent of inducible nitric oxide synthase immunopositivity (mean \pm standard error of the mean [SEM]) in the studied groups

\begin{tabular}{lcccc}
\hline & Group I (Control) & Group II (SIM) & Group III (ISO) & Group IV (ISO/SIM) \\
\hline Mean \pm SEM & $22.477 \pm 2.101$ & $19.379 \pm 1.706$ & $66.653 \pm 3.220$ & $34.741 \pm 1.410$ \\
P1 & & 1.000 & $0.000^{*}$ & $0.007^{*}$ \\
P2 & 1.000 & & $0.000^{*}$ & $0.001^{*}$ \\
P3 & $0.000^{*}$ & $0.000^{*}$ & & $0.000^{*}$ \\
\hline
\end{tabular}

P1 vs. group I; P2 vs. group II; P3 vs. group III; *significant difference $(\mathrm{p} \leq 0.05)$

ing for iNOS of these sections was undetectable in both control rats and rats treated with SIM alone (Fig. 4A, B). On the other hand, ISO injection induced significant iNOS immunostaining which was primarily localised in sarcoplasm of cardiomyocytes (Fig. 4C) with some immunostaining noted within the inflammatory cells. Co-administration of SIM with ISO induced depletion of iNOS immunostaining in sarcoplasm of cardiomyocytes (Table 9, Fig. 4D).

Heart sections immunostained with anti-thioredoxin antibody expressed moderate immunoreactivity in the sarcoplasm of cardiomyocytes in control rats and SIM-treated rats (Fig. 5A, B). ISO injection demonstrated strong immunoreactivity for thioredoxin in the sarcoplasm and almost all nuclei of cardiomyocytes (Fig. 5C). Co-administration of SIM with ISO induced moderate thioredoxin immunoreactivity in the sarcoplasm of cardiomyocytes (Table 10, Fig. 5D).

\section{DISCUSSION}

Intraperitoneal administration of ISO for 7 days in adult male rats produced cardiac hypertrophy, increased HW/BW ratio, and fibrosis as evidenced by increased expression of collagen types I and III. These histopathological changes were associated with significant increase in serum levels of both cardiac injury markers, namely troponin-T and CPK-MB, and pro-inflammatory biomarkers, namely TNF- $\alpha$ and IL-6. Also, immunohistochemistry demonstrated increased iNOS and thioredoxin immunoreactivity in cardiomyocytes in ISO-injected rats. Co-administration of SIM markedly attenuated the ISO-induced histopathological alterations. The increased immunoexpression of iNOS in cardiomyocytes in ISO-injected rats and the marked diminution observed in rats simultaneously treated with SIM supports the key role of inhibiting iNOS in attenuating the deleterious cardiac effects of ISO injection. 


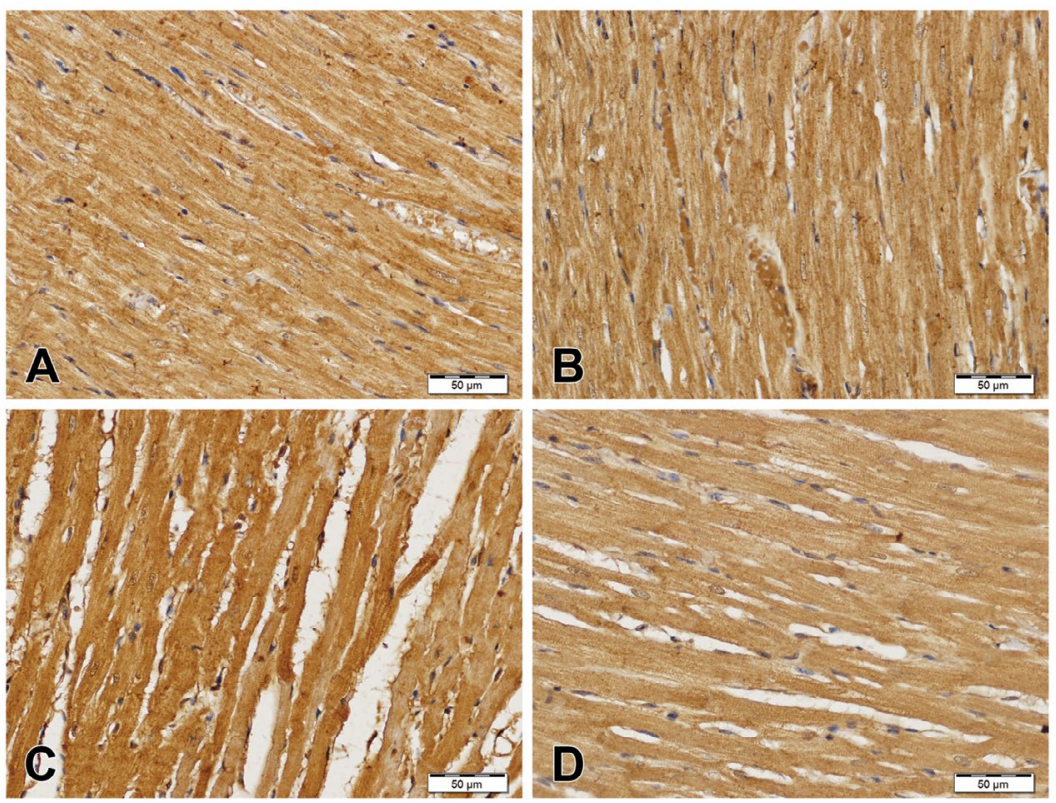

Figure 5. Heart sections immunostained with anti-thioredoxin antibody. From control rat (A), and from SIM group (B) showing moderate immunoreactivity; C. From ISO group showing strong immunoreactivity; D. From ISO/SIM group showing moderate immunoreactivity. Scale bars $=50 \mu \mathrm{m}$.

Table 10. Area per cent of thioredoxin immunopositivity (mean \pm standard error of the mean [SEM]) in the studied groups

\begin{tabular}{lcccc}
\hline & Group I (Control) & Group II (SIM) & Group III (ISO) & Group IV (IS0/SIM) \\
\hline Mean \pm SEM & $51.386 \pm 3.337$ & $50.927 \pm 3.330$ & $78.961 \pm 1.417$ & $66.452 \pm 2.628$ \\
P1 & & 1.000 & $0.000^{*}$ & $0.012^{*}$ \\
P2 & 1.000 & & $0.000^{*}$ & $0.010^{*}$ \\
P3 & $0.000^{*}$ & $0.000^{*}$ & & $0.045^{*}$ \\
\hline
\end{tabular}

P1 vs. group I; P2 vs. group II; P3 vs. group III; *significant difference $(p \leq 0.05)$

Previous study had shown that repeated ISO administration affords a simple non-invasive means for inducing myocardial injury and diastolic dysfunction with a low incidence of morality [7]. It was proved that a single injection of ISO results in a rapid, marked increase in myocardial necrosis in rats [42]. Another study demonstrated that the extent of myocardial lesions in rat heart could be increased with repeated ISO injections [53].

A significant increase of $\mathrm{HW} / \mathrm{BW}$ ratio induced by ISO injection as compared with control rats is considered as a sensitive indicator of cardiac hypertrophy [15, $58,60]$. Co-administration of SIM significantly reduced $\mathrm{HW}$ and HW/BW ratio in ISO-injected rats. Previous studies showed that chronic administration of ISO produced LVH $[9,28,45,52]$ and increased ventricular collagen content [4]. In this regard, experimental data are accumulating, suggesting that the release of superoxide $\left(\mathrm{O}_{2}^{-}\right)$induces cardiomyocyte hypertrophy [51].
Determination of collagen type I by Masson's trichrome and collagen type III by immunohistochemical staining suggested marked increase of collagen content in the LV of ISO-injected rats relative to controls. Increased collagen deposition in the ventricular extracellular matrix is considered to be a sensitive indicator of fibrosis development [11]. A previous study in rats proved that expression of collagen types I and III mRNA increased after ISO administration and extracellular matrix transcripts are increased in the LV with ISO administration [5, 34]. Similarly, other studies [1, 12, 24] indicated that collagen volume fraction of the hypertrophied LV was significantly increased as a result of experimental hypertension.

Isoproterenol-injected rats showed elevated serum levels of TNF- $\alpha$ and IL- 6 . It has been proposed that ROS and pro-inflammatory cytokines are playing a critical 
role in LV remodelling. Previous studies have showed that $\beta$-adrenergic stimulation with ISO-induced cardiac expression of proinflammatory cytokines such as TNF- $\alpha$ and IL-6 $[33,37]$ was associated with increased ROS production and imbalance between nitric acid and superoxide anion and these changes could contribute to the structural and functional alterations observed [33]. Choudhary et al. [9] stated that ISO administration resulted not only in cardiac hypertrophy and apoptosis, but also increased oxidative stress in ventricular tissue as evidenced by increased ROS contents.

Increased expression of iNOS in cytosol of cardiomyocytes induced by ISO administration was demonstrated. It was proved that ISO administration increased iNOS producing greater amounts of NO [27, 30]. iNOS is expressed at low levels in cytosol of normal cardiomyocytes but could be induced in response to several stimuli such as tissue injury, inflammation, cytokines, and growth factors as well as cardiovascular disease $[10,31,49,61]$. Overexpression of iNOS causes cardiomyopathy, heart block, and sudden death [36]. The detrimental cardiac effects of iNOS have been documented by many other investigators $[10,20]$. The produced NO reacts with superoxide anion $\left(\mathrm{O}_{2}^{-}\right)$to form the highly reactive oxidant peroxynitrite ( $\left.\mathrm{ONOO}^{-}\right)$ [54]. Peroxynitrite can nitrate proteins, which can lead to protein dysfunction [54]. Beckman and Koppenol [3] stated that the detrimental effects of peroxynitrite include oxidation of lipids, nitration of protein, oxidation of free protein-associated thiols and stimulation of apoptosis. Another study demonstrated that peroxynitrite is a major contributor to cytokine-induced myocardial dysfunction [14]. iNOS is also capable of generating $\mathrm{O}_{2}^{-}$independent of NO production [59]. $\mathrm{O}_{2}^{-}$can also dismutate, yielding hydrogen peroxide, which can be reduced to highly reactive hydrogen radicals. These preoxidants promote lipid peroxidation, damaging the heart and blood vessels $[36,55]$. Song et al. [47] have demonstrated that iNOS expression induces apoptosis in cardiomyocytes.

Co-treatment with SIM effectively attenuated the ISO-induced cardiac changes including cardiac hypertrophy, fibrosis, inflammation, and oxidative stress in adult male rats. SIM effectively prevented the hypertrophic growth as evidenced by the normal level of HW/BW ratio in the SIM co-treated rats compared with controls. In accordance, previous studies indicated that treatment with SIM markedly attenuated the myocardial hypertrophy of pressure-overloaded hearts $[22,38,51]$. This could be attributed to the antioxidant property of SIM [51] as evidenced by the marked depletion of iNOS in cardiomyocytes.

Heart sections from ISO-injected rats showed a significant increase in the mean area per cent of strong immunoreaction for thioredoxin compared to control rats. It has been reported that enhanced thioredoxin expression may be induced by ROS produced by infiltrating inflammatory cells in acute myocarditis [2]. Administration of SIM significantly reduced ISO-induced thioredoxin expression. Thus, the observed cardioprotective effect of SIM was probably attributed to the presence of antioxidant and anti-inflammatory properties.

\section{Clinical implications}

The present study supports the cardioprotective role of SIM co-administration against ISO-induced LVH through inhibition of iNOS expression as well as attenuation of pro-fibrotic and pro-inflammatory cytokines, namely TNF- $\alpha$ and IL-6. Also, data concerning the anti-hypertrophic signalling pathways will allow the evolution of new approaches for therapy of pathological LVH.

\section{Limitations of the study}

Microscopic examination of heart sections is considered the most reliable method for detecting myocardial fibrosis. However, the usage of non-invasive methods to demonstrate the presence of myocardial fibrosis in rats with spontaneous hypertension and in patients with essential hypertension would be highly beneficial. In this aspect, the present study did not apply a biochemical assessment of fibrillar collagen synthesis and degradation. Also, this study did not assess the changes in expression of other types of NOS including both eNOS and nNOS.

\section{CONCLUSIONS}

The present results demonstrated that cardiac hypertrophy, fibrosis, inflammation, and oxidation produced by 7 days of ISO injections could be substantially attenuated by SIM co-administration. These results are also supported by the detected alterations in the immunostaining of iNOS in cardiomyocytes which was increased by ISO injection and depleted by SIM co-treatment. Deletion of iNOS reduces ROS production and oxidative stress, which may be responsible for the beneficial cardiac effect observed by SIM co-treatment. 


\section{Acknowledgements}

The author gratefully acknowledges the Research Centre, College of Medicine, Deanship of Scientific Research, King Saud University, Riyadh, Kingdom of Saudi Arabia for the financial support and continuous encouragement.

\section{REFERENCES}

1. Abrahams C, Janicki JS, Weber KT. Myocardial hypertrophy in Macaca fascicularis. Structural remodeling of the collagen matrix. Lab Invest. 1987; 56(6): 676-683, indexed in Pubmed: 2955170.

2. Arnér ES, Holmgren A. Physiological functions of thioredoxin and thioredoxin reductase. Eur J Biochem. 2000; 267(20): 6102-6109, doi:10.1046/j.14321327.2000.01701.x, indexed in Pubmed: 11012661.

3. Beckman JS, Koppenol WH. Nitric oxide, superoxide, and peroxynitrite: the good, the bad, and ugly. Am J Physiol. 1996; 271(5 Pt 1): C1424-C1437, doi:10.1136/ thx.2004.038471, indexed in Pubmed: 8944624.

4. Benjamin IJ, Jalil JE, Tan LB, et al. Isoproterenol-induced myocardial fibrosis in relation to myocyte necrosis. Circ Res. 1989; 65(3): 657-670, doi:10.1161/01.res.65.3.657, indexed in Pubmed: 2527639.

5. Boluyt MO, Long $X$, Eschenhagen $T$, et al. Isoproterenol infusion induces alterations in expression of hypertrophyassociated genes in rat heart. Am J Physiol. 1995; 269(2 Pt 2): H638-H647, indexed in Pubmed: 7653628.

6. Bradbury P, Rae K. Connective tissues and stains. In: Bancroft J, Stevens A, editors. Theory and Practice of Histological Techqniues. 4th ed. Churchill Livingstone, London, New York, Toronto. 1999: pp. 113-138.

7. Brooks WW, Conrad CH. Isoproterenol-induced myocardial injury and diastolic dysfunction in mice: structural and functional correlates. Comp Med. 2009; 59(4): 339-343, indexed in Pubmed: 19712573.

8. Chen YQ, Zhao LY, Zhang WZ, et al. Simvastatin reverses cardiomyocyte hypertrophy via the upregulation of phosphatase and tensin homolog expression. Exp Ther Med. 2015; 10(2): 797-803, doi: 10.3892/etm.2015.2550, indexed in Pubmed: 26622396.

9. Choudhary R, Mishra KP, Subramanyam C. Prevention of isoproterenol-induced cardiac hypertrophy by eugenol, an antioxidant. Indian J Clin Biochem. 2006; 21(2): 107-113, doi: 10.1007/BF02912923, indexed in Pubmed: 23105625.

10. Csont T, Viappiani S, Sawicka J, et al. The involvement of superoxide and iNOS-derived NO in cardiac dysfunction induced by pro-inflammatory cytokines. J Mol Cell Cardiol. 2005; 39(5): 833-840, doi: 10.1016/j.yjmcc.2005.07.010, indexed in Pubmed: 16171809.

11. D'Armiento J. Matrix metalloproteinase disruption of the extracellular matrix and cardiac dysfunction. Trends Cardiovasc. Med. 2002; 12(3): 97-101, doi: 10.1016/ s1050-1738(01)00160-8, indexed in Pubmed: 12007733.

12. Doering CW, Jali JE, Janicki JS, et al. Collagen network remodelling and diastolic stiffness of the rat left ventricle with pressure overload hypertrophy. Cardiovasc Res. 1988; 22(10): 686-695, doi: 10.1093/cvr/22.10.686, indexed in Pubmed: 2978464.
13. Escames G, Khaldy H, León J, et al. Changes in iNOS activity, oxidative stress and melatonin levels in hypertensive patients treated with lacidipine. J Hypertens. 2004; 22(3): 629-635, doi: 10.1097/00004872-200403000-00027, indexed in Pubmed: 15076170.

14. Ferdinandy P, Danial H, Ambrus I, et al. Peroxynitrite is a major contributor to cytokine-induced myocardial contractile failure. Circ Res. 2000; 87(3): 241-247, doi: 10.1161/01. res.87.3.241, indexed in Pubmed: 10926876.

15. Gealekman O, Abassi Z, Rubinstein I, et al. Role of myocardial inducible nitric oxide synthase in contractile dysfunction and beta-adrenergic hyporesponsiveness in rats with experimental volume-overload heart failure. Circulation. 2002; 105(2): 236-243, doi: 10.1161/hc0202.102015, indexed in Pubmed: 11790707.

16. Gradman AH, Alfayoumi F. From left ventricular hypertrophy to congestive heart failure: management of hypertensive heart disease. Prog Cardiovasc Dis. 2006; 48(5): 326-341, doi: 10.1016/j.pcad.2006.02.001, indexed in Pubmed: 16627048.

17. Guzy PM. Creatine phosphokinase-MB (CPK-MB) and the diagnosis of myocardial infarction. West J Med. 1977; 127(6): 455-460, doi:10.1056/nejm199503023320914, indexed in Pubmed: 339548.

18. Habib FM, Springall DR, Davies GJ, et al. Tumour necrosis factor and inducible nitric oxide synthase in dilated cardiomyopathy. Lancet. 1996; 347(9009): 1151-1155, doi: 10.1016/s0140-6736(96)90610-8, indexed in Pubmed: 8609750.

19. Haywood GA, Tsao PS, von der Leyen HE, et al. Expression of inducible nitric oxide synthase in human heart failure. Circulation. 1996; 93(6): 1087-1094, doi: 10.1161/01. cir.93.6.1087, indexed in Pubmed: 8653828.

20. Henderson BC, Tyagi N, Ovechkin A, et al. Oxidative remodeling in pressure overload induced chronic heart failure. Eur J Heart Fail. 2007; 9(5): 450-457, doi: 10.1016/j. ejheart.2006.12.008, indexed in Pubmed: 17306621.

21. Huang $X Y$, Chen $C X$, Zhang $X M$, et al. Effects of ethanolic extract from Radix Scrophulariae on ventricular remodeling in rats. Phytomedicine. 2012; 19(3-4): 193-205, doi: 10.1016/j.phymed.2011.09.079, indexed in Pubmed: 22035768.

22. Indolfi C, Di Lorenzo E, Perrino C, et al. Hydroxymethylglutaryl coenzyme A reductase inhibitor simvastatin prevents cardiac hypertrophy induced by pressure overload and inhibits p21ras activation. Circulation. 2002; 106(16): 2118-2124, doi: 10.1161/01.cir.0000034047.70205.97, indexed in Pubmed: 12379583.

23. Jaeschke H. Mechanisms of oxidant stress-induced acute tissue injury. Proc Soc Exp Biol Med. 1995; 209(2): 104-111, doi: 10.3181/00379727-209-43885b, indexed in Pubmed: 7770460.

24. Jalil JE, Doering CW, Janicki JS, et al. Fibrillar collagen and myocardial stiffness in the intact hypertrophied rat left ventricle. Circ Res. 1989; 64(6): 1041-1050, doi: 10.1161/01. res.64.6.1041, indexed in Pubmed: 2524288.

25. Kaden J. IL-6 determination in serum of kidney graft recipients by a new bedside test: its diagnostic relevance. Transplant Proc. 2007; 39(2): 511-513, doi: 10.1016/j.transproceed.2006.12.015, indexed in Pubmed: 17362770. 
26. Kapur NK, Musunuru K. Clinical efficacy and safety of statins in managing cardiovascular risk. Vasc Health Risk Manag. 2008; 4(2): 341-353, doi:10.2147/vhrm.s1653, indexed in Pubmed: 18561510.

27. Kelly RA, Balligand JL, Smith TW. Nitric oxide and cardiac function. Circ. Res. 1996; 79(3): 363-380, doi: 10.1161/01. res.79.3.363, indexed in Pubmed:8781470.

28. Leenen FH. Cardiovascular consequences of sympathetic hyperactivity. Can J Cardiol. 1999; 15 Suppl A: 2A-7A, indexed in Pubmed: 10205250.

29. Lefer DJ, Granger DN. Oxidative stress and cardiac disease. Am J Med. 2000; 109(4): 315-323, doi: 10.1016/s00029343(00)00467-8, indexed in Pubmed: 10996583.

30. Lin $Y$, Wang LN, Xi YH, et al. L-arginine inhibits isoproterenol-induced cardiac hypertrophy through nitric oxide and polyamine pathways. Basic Clin Pharmacol Toxicol. 2008; 103(2): 124-130, doi: 10.1111/j.17427843.2008.00261.x, indexed in Pubmed: 18816294.

31. Liu YH, Carretero OA, Cingolani OH, et al. Role of inducible nitric oxide synthase in cardiac function and remodeling in mice with heart failure due to myocardial infarction. Am J Physiol Heart Circ Physiol. 2005; 289(6): H2616-H2623, doi: 10.1152/ajpheart.00546.2005, indexed in Pubmed: 16055518.

32. Lloyd-Jones DM, Larson MG, Leip EP, et al. Framingham Heart Study. Lifetime risk for developing congestive heart failure: the Framingham Heart Study. Circulation. 2002; 106(24): 3068-3072, doi: 10.1161/01. cir.0000039105.49749.6f, indexed in Pubmed: 12473553.

33. Martín-Fernández $B$, de las Heras $N$, Miana $M$, et al. Spironolactone prevents alterations associated with cardiac hypertrophy produced by isoproterenol in rats: involvement of serum- and glucocorticoid-regulated kinase type 1. Exp Physiol. 2012; 97(6): 710-718, doi:10.1113/ expphysiol.2011.063230, indexed in Pubmed: 22327331.

34. Masson S, Arosio B, Luvarà G, et al. Remodelling of cardiac extracellular matrix during beta-adrenergic stimulation: upregulation of SPARC in the myocardium of adult rats. J Mol Cell Cardiol. 1998; 30(8): 1505-1514, doi: 10.1006/ jmcc.1998.0714, indexed in Pubmed: 9737937.

35. McKinsey TA, Kass DA. Small-molecule therapies for cardiac hypertrophy: moving beneath the cell surface. Nat Rev Drug Discov. 2007; 6(8): 617-635, doi: 10.1038/nrd2193, indexed in Pubmed: 17643091.

36. Mungrue IN, Gros R, You X, et al. Cardiomyocyte overexpression of iNOS in mice results in peroxynitrite generation, heart block, and sudden death. J Clin Invest. 2002; 109(6): 735-743, doi: 10.1172/JCl13265, indexed in Pubmed: 11901182.

37. Murray DR, Prabhu SD, Chandrasekar B. Chronic beta-adrenergic stimulation induces myocardial proinflammatory cytokine expression. Circulation. 2000; 101(20): 2338-2341, doi: 10.1161/01.cir.101.20.2338, indexed in Pubmed: 10821806.

38. Nadruz W, Lagosta VJ, Moreno H, et al. Simvastatin prevents load-induced protein tyrosine nitration in overloaded hearts. Hypertension. 2004; 43(5): 1060-1066, doi: 10.1161/01.HYP.0000124252.43470.2c, indexed in Pubmed: 15023931.
39. Narang D, Sood S, Thomas M, et al. Dietary palm olein oil augments cardiac antioxidant enzymes and protects against isoproterenol-induced myocardial necrosis in rats. J Pharm Pharmacol. 2005; 57(11): 1445-1451, doi: 10.1211/jpp.57.11.0010, indexed in Pubmed: 16259777.

40. Porter KE, Turner NA. Cardiac fibroblasts: at the heart of myocardial remodeling. Pharmacol Ther. 2009; 123(2): 255-278, doi:10.1016/j.pharmthera.2009.05.002, indexed in Pubmed: 19460403.

41. Querejeta R, López B, González A, et al. Increased collagen type I synthesis in patients with heart failure of hypertensive origin: relation to myocardial fibrosis. Circulation. 2004; 110(10): 1263-1268, doi: 10.1161/01.CIR.0000140973.60992.9A, indexed in Pubmed: 15313958.

42. RONA G, CHAPPEL Cl, BALAZS T, et al. An infarct-like myocardial lesion and other toxic manifestations produced by isoproterenol in the rat. AMA Arch Pathol. 1959; 67(4): 443-455, indexed in Pubmed: 13636626.

43. Rona G. Catecholamine cardiotoxicity. J Mol Cell Cardiol. 1985; 17(4): 291-306, doi: 10.1016/s00222828(85)80130-9, indexed in Pubmed: 3894676.

44. Shah AM, MacCarthy PA. Paracrine and autocrine effects of nitric oxide on myocardial function. Pharmacol Ther. 2000; 86(1): 49-86, doi:10.1016/s0163-7258(99)00072-8, indexed in Pubmed: 10760546.

45. Shizukuda Y, Buttrick PM, Geenen DL, et al. beta-adrenergic stimulation causes cardiocyte apoptosis: influence of tachycardia and hypertrophy. Am J Physiol. 1998; 275(3 Pt 2): H961-H968, indexed in Pubmed: 9724301.

46. Siwik DA, Pagano PJ, Colucci WS. Oxidative stress regulates collagen synthesis and matrix metalloproteinase activity in cardiac fibroblasts. Am J Physiol Cell Physiol. 2001; 280(1): C53-C60, indexed in Pubmed: 11121376.

47. Song W, Lu X, Feng Q. Tumor necrosis factor-alpha induces apoptosis via inducible nitric oxide synthase in neonatal mouse cardiomyocytes. Cardiovasc Res. 2000; 45(3): 595-602, doi: 10.1016/s0008-6363(99)00395-8, indexed in Pubmed: 10728381.

48. Stevens A, Wilson I. The haematoxylins and eosin. In: Bancroft J, editors. Theory and Practice of Histological Techqniues. 4th ed. Churchill Livingstone, London, New York, Toronto. 1999: pp. 99-112.

49. Sun Y, Carretero OA, Xu J, et al. Lack of inducible NO synthase reduces oxidative stress and enhances cardiac response to isoproterenol in mice with deoxycorticosterone acetate-salt hypertension. Hypertension. 2005; 46(6): 1355-1361, doi: 10.1161/01.HYP.0000192651.06674.3f, indexed in Pubmed: 16286571.

50. Swan HJ. Left ventricular dysfunction in ischemic heart disease: fundamental importance of the fibrous matrix. Cardiovasc Drugs Ther. 1994; 8 Suppl 2: 305-312, doi: 10.1007/bf00877314, indexed in Pubmed: 7947372.

51. Takemoto M, Node K, Nakagami H, et al. Statins as antioxidant therapy for preventing cardiac myocyte hypertrophy. J Clin Invest. 2001; 108(10): 1429-1437, doi: 10.1172/ JCl13350, indexed in Pubmed: 11714734. 
52. Taylor PB, Tang Q. Development of isoproterenol-induced cardiac hypertrophy. Can J Physiol Pharmacol. 1984; 62(4): 384-389, doi: 10.1139/y84-191, indexed in Pubmed: 6203632 .

53. Teerlink JR, Pfeffer JM, Pfeffer MA. Progressive ventricular remodeling in response to diffuse isoproterenol-induced myocardial necrosis in rats. Circ Res. 1994; 75(1): 105-113, doi: 10.1161/01 res.75.1.105, indexed in Pubmed: 8013068.

54. Torres SH, De Sanctis JB, de L Briceño M, et al. Inflammation and nitric oxide production in skeletal muscle of type 2 diabetic patients. J Endocrinol. 2004; 181(3): 419-427, doi: 10.1677/joe.0.1810419, indexed in Pubmed: 15171690.

55. Touyz RM. Reactive oxygen species, vascular oxidative stress, and redox signaling in hypertension: what is the clinical significance? Hypertension. 2004; 44(3): 248-252, doi: 10.1161/01.HYP.0000138070.47616.9d, indexed in Pubmed: 15262903.

56. Vasan RS, Levy D. The role of hypertension in the pathogenesis of heart failure. A clinical mechanistic overview. Arch Intern Med. 1996; 156(16): 1789-1796, doi: 10.1001/ archinte.156.16.1789, indexed in Pubmed: 8790072.

57. Wan LH, Chen J, Li L, et al. Protective effects of Carthamus tinctorius injection on isoprenaline-induced myocardial injury in rats. Pharm Biol. 2011; 49(11): 1204-1209, do i: 10.3109/13880209.2011.576348, indexed in Pubmed: 22014268.

58. Warren CM, Jordan MC, Roos KP, et al. Titin isoform expression in normal and hypertensive myocardium. Cardiovasc Res. 2003; 59(1): 86-94, doi:10.1016/s00086363(03)00328-6, indexed in Pubmed: 12829179.

59. Xia Y, Zweier JL. Superoxide and peroxynitrite generation from inducible nitric oxide synthase in macrophages. Proc Natl Acad Sci USA. 1997; 94(13): 6954-6958, doi: 10.1073/ pnas.94.13.6954, indexed in Pubmed: 9192673.

60. Yang J, Wang HX, Zhang YJ, et al. Astragaloside IV attenuates inflammatory cytokines by inhibiting TLR4/NF- $\kappa$ B signaling pathway in isoproterenol-induced myocardial hypertrophy. J Ethnopharmacol. 2013 [Epub ahead of print], doi: 10.1016/j.jep.2013.10.017, indexed in Pubmed: 24513635.

61. Zhang $P, X u X, H u X$, et al. Inducible nitric oxide synthase deficiency protects the heart from systolic overload-induced ventricular hypertrophy and congestive heart failure. Circ Res. 2007; 100(7): 1089-1098, doi: 10.1161/01.RES.0000264081.78659.45, indexed in Pubmed: 17363700. 